\title{
Newer Is Not Always Better: All Antihypertensive Medications Do Not Equally Reduce Cardiovascular Risk
}

\author{
Gerald W. Smetana, MD \\ Division of General Medicine and Primary Care, Beth Israel Deaconess Medical Center and Harvard Medical School, Boston, MA, USA.
}

J Gen Intern Med 27(6):618-20

DOI: $10.1007 / \mathrm{s} 11606-012-2020-\mathrm{x}$

(c) Society of General Internal Medicine 2012

$\mathrm{H}$ ypertension contributes more to cardiovascular mortality than any other modifiable risk factor including cigarette use, elevated LDL cholesterol, obesity, and diabetes. ${ }^{1}$ Forty-five percent of all cardiovascular deaths are attributable to hypertension. In addition, hypertension is a major risk factor for both stroke and myocardial infarction. In large part due to rising obesity rates, the prevalence of hypertension in the U.S. is increasing. According to the NHANES data, from the early 1990s to the most recently available data from 2005-2008, the prevalence of hypertension has risen from $25.5 \%$ to $30.9 \%{ }^{2}$ The majority of the U.S. elderly are hypertensive: the prevalence is $80 \%$ of woman and $67 \%$ of men aged 75 years or older. Given the pervasiveness and impact of hypertension, it is critically important to assess the effect of various antihypertensive medications on cardiovascular morbidity. Most studies of antihypertensive medication report proxy outcomes, commonly blood pressure reduction, rather than cardiovascular morbidity, and are of relatively brief duration. Two important questions for clinicians are: 1) Does the degree of blood pressure reduction correspond to the magnitude of cardiovascular risk reduction? and 2) Do all antihypertensive medications equally reduce cardiovascular risk?

Thiazide diuretics, in particular chlorthalidone, are the antihypertensive drug class with the most robust supporting evidence. JNC-7, a guideline published by the National Heart, Lung, and Blood Institute (NHLBI) in 2003, recommends diuretics as the preferred first line antihypertensive agent, unless a compelling reason exists to select an alternative drug. ${ }^{3}$ A discussion of the basis for this recommendation goes beyond the scope of this editorial. Not all guidelines have endorsed this approach; other agencies have concluded that all medications that lower blood pressure are equally effective in their ability to reduce cardiovascular risk. For example, the European Society of

Published online March 2, 2012
Hypertension guidelines consider all drugs classes to be comparable ${ }^{4}$ and the Australian guidelines state that all drug classes are equivalent with the exception of beta blockers. ${ }^{5}$ The Australian guidelines go further in limiting the first line use of thiazide diuretics to the elderly.

In fact, over the past decade in particular, a growing body of literature has suggested that all antihypertensive medications do not equally reduce cardiovascular risk. In the landmark ALLHAT study, reported in 2002, the doxazosin arm was terminated early due to higher cardiovascular events, in particular a doubling of the congestive heart failure rates, when compared to chlorthalidone. ${ }^{6}$ Since that report, most editorialists and hypertension experts have recommended that alpha blockers no longer be used for the initial treatment of hypertension. ${ }^{7,8}$ Subsequently, several meta-analyses have re-examined the value of beta blockers in the treatment of hypertension. ${ }^{9-11}$ In these reviews, beta blockers were inferior to other antihypertensive agents. In fact, in the Cochrane review, beta blockers were no more effective than placebo for the outcomes of coronary heart disease events, cardiovascular mortality, and total mortality. ${ }^{11}$ Despite their widespread use over several decades, beta blockers should no longer be used as initial or secondary treatment for hypertension (indications other than hypertension remain).

In a 2009 ambitious Cochrane Collaboration review of the entire body of literature of placebo controlled randomized trials of first line therapy for mild hypertension, only thiazides and ACE inhibitors reduced all four clinical endpoints (mortality, stroke, coronary artery disease, and total cardiovascular events). ${ }^{12}$ There were no eligible trials of angiotensin receptor blockers (ARBs).

Among the potential options for initial treatment of hypertension are drugs that work through the reninangiotensin-aldosterone system: ACE inhibitors, ARBs, and a renin inhibitor (aliskiren). Until recently, cost has been an important barrier to the use of ARBs. However, with the availability of generic losartan, and the upcoming expiration of the patent for valsartan in September 2012, it is timely to consider the relative benefit of ARBs and ACE inhibitors in the initial management of hypertension.

In this issue of the Journal, Powers and colleagues report an updated analysis of the comparative effectiveness of ACE inhibitors, ARBs, and a renin inhibitor. ${ }^{13}$ This work 
derived from a review that was commissioned by the Agency for Healthcare Quality and Research (AHRQ). ${ }^{14}$ They conducted a systematic review of articles of at least 20 weeks in duration that directly compared one of these classes to another and reported at least one of several proxy, tolerability, or clinical outcomes. The authors did not include trials of a drug compared only to placebo. Among 110 articles (100 discrete studies), only two evaluated the direct renin inhibitor aliskiren; neither reported cardiovascular endpoint data. Among the remaining studies that compared ACE inhibitors to ARBs, most (58 of 77 studies) reported no difference in blood pressure reduction. Twentysix studies provided data on successful blood pressure control with a single agent; there was no difference between the two drug classes (pooled OR 1.08, 95\% CI 0.94-1.25). As expected, cough was more common among subjects receiving an ACE inhibitor (OR 4.74, 95\% CI 3.56-6.31); withdrawals to adverse events were marginally more common for ACE inhibitors (5.3\% vs. 3\%). Subgroup analysis was possible for a small number of studies; the authors indicate that results for women, the elderly, AfricanAmericans, and those with diabetes, the outcomes did not differ substantially from the overall results.

Importantly, data on cardiovascular outcomes were limited. Most of the studies did not enroll high risk patients and few cardiovascular events occurred. While 21 studies of 38,589 subjects reported rates of myocardial infarction, stroke, or death, the overall event rates were too low to draw meaningful conclusions: only 38 deaths and 13 strokes occurred.

This commendable systematic review by Powers and colleagues was rigorous, well designed, and conducted in accordance with current recommended standards. ${ }^{15,16}$ However, several important issues limit the generalizability of these results for decision making in clinical practice. Due to limitations in the source data, this review primarily assesses proxy outcomes. In addition, most studies excluded high risk patients and were of a duration that would be too brief to demonstrate meaningful differences in cardiovascular event rates (median follow-up was 24 weeks).

While ACEi and ARB reduce blood pressure equally, whether they equally reduce cardiovascular risk remains uncertain. In two early trials of ARB monotherapy for primary prevention that evaluated cardiovascular outcomes, losartan was more effective than atenolol (LIFE), ${ }^{17}$ while valsartan and amlodipine comparably reduced risk (VALUE). ${ }^{18}$ The LIFE trial was, however, flawed by virtue of selecting an inferior drug as the comparator. ${ }^{17}$ One of the largest comparative studies $(n=17,044)$ to evaluate cardiovascular outcomes, the ONTARGET trial, was excluded from the current review due to the lack of subgroup analysis of patients with hypertension. ${ }^{19}$ In this trial of patients with existing cardiovascular disease or diabetes, monotherapy with telmisartan reduced cardiovascular events to the same extent as an ACE inhibitor, ramipril, over a mean follow up of 4.7 years. This provides support for equivalence in the important endpoint of cardiovascular outcomes (however dual therapy with both drugs did not further reduce cardiovascular event rates and led to more adverse events).

In contrast, several recent large secondary prevention studies of ARB versus placebo (therefore ineligible for this review) have raised concerns about the efficacy and safety of ARBs. In the TRANSCEND trial of telmisartan versus placebo in patients with existing cardiovascular disease or diabetes who were ACE inhibitor intolerant (a common clinical scenario), there was no difference in the primary outcome after a mean follow up of 4.7 years. $^{20}$ After adjustment, there was also no difference in any of the secondary clinical outcomes. ARBs were no more effective than placebo. Similarly, in the NAVIGATOR trial of patients with glucose intolerance $(77 \%$ of whom were hypertensive), valsartan did not reduce cardiovascular events compared to placebo $^{21}$ and in PRoFESS, 2.5 years of telmisartan did not reduce stroke rates compared to placebo among patients with a recent stroke. ${ }^{22}$ In a 2011 systematic review of studies of ARBs versus placebo that evaluated cardiovascular outcomes, ARBs did not reduce mortality, cardiovascular mortality, or rates of myocardial infarction. $^{23}$ ARBs reduced stroke rates (OR 0.91, 95\% CI 0.85-0.98), but this effect is less than is seen with other antihypertensive drug classes. ${ }^{12}$ This review, however, included trials for indications other than hypertension (e.g. heart failure, diabetes) and did not report the hypertension results separately.

In the only reported comparative efficacy study of aliskiren (renin inhibitor) that evaluated cardiovascular outcomes, the ALTITUDE trial of patients with diabetes and renal impairment, the addition of aliskiren to an ACE inhibitor or ARB actually increased risk of stroke or adverse events; the study was terminated early based on recommendations from the independent data monitoring committee that was overseeing the study. ${ }^{24}$ Newer is not necessarily better.

The review of Powers and colleagues establishes that ACE inhibitors and ARBs comparably reduce blood pressure. Both drug classes probably reduce cardiovascular risk equally for primary prevention; however ARBs are ineffective for secondary prevention of cardiovascular disease. Even as cost considerations become less of a factor in clinical decision making, thiazide diuretics are the preferred choices for initial therapy of hypertension; ACE inhibitors, and calcium channel blockers are also acceptable first line options. Among ACE intolerant patients, I would advise against the use of ARBs for monotherapy based on the currently available evidence. This is an extrapolation from the secondary prevention trials; however, other classes of antihypertensive medications more unambiguously reduce cardiovascular endpoints. Current data do not support 
the use of aliskiren; the only available cardiovascular outcome data suggest harm. While ARBs cause less cough and are marginally better tolerated than ACE inhibitors, this observation alone is not sufficient to elevate the role of ARBs in initial treatment of hypertension. The NHLBI expects to release JNC-8 in 2012, potentially coincident with this editorial. The guideline authors must endeavor to incorporate recent trial data and an important observation: all drugs that lower blood pressure do not equally reduce cardiovascular risk.

Acknowledgements: Dr. Smetana has served as a consultant to Anvita Health.

Corresponding Author: Gerald W. Smetana, MD; Division of General Medicine and Primary Care, Beth Israel Deaconess Medical Center and Harvard Medical School, Shapiro 621D, 330 Brookline Ave., Boston, MA 02215, USA (e-mail: gsmetana@bidmc.harvard. edu).

\section{REFERENCES}

1. Danaei G, Ding E, Mozaffarian D, et al. The Preventable Causes of Death in the United States: Comparative Risk Assessment of Dietary, Lifestyle, and Metabolic Risk Factors. PLoS Med 2009;6:e1000058. doi:10.1371/journal.pmed.

2. National Center for Health Statistics. Health, United States 2010: with special feature on death and dying. Hyattsville, MD. 2011.

3. Chobanian AV, Bakris GL, Black HR, et al. The Seventh Report of the Joint National Committee on Prevention, Detection, Evaluation, and Treatment of High Blood Pressure: the JNC 7 report. JAMA 2003;289:2560-72.

4. Mancia G, Laurent S, Agabiti-Rosei E, et al. Reappraisal of European guidelines on hypertension management: a European Society of Hypertension Task Force document. Blood Press 2009;18:308-47.

5. National Heart Foundation of Australia (National Blood Pressure and Vascular Disease Advisory Committee). Guide to management of hypertension 2008. Updated December 2010. Available at: www. heartfoundation.org.au

6. ALLHAT Collaborative Research Group. Major cardiovascular events in hypertensive patients randomized to doxazosin vs chlorthalidone: the antihypertensive and lipid-lowering treatment to prevent heart attack trial (ALLHAT). JAMA 2000;283:1967-75.

7. Messerli FH. Implications of discontinuation of doxazosin arm of ALLHAT. Antihypertensive and Lipid-Lowering Treatment to Prevent Heart Attack Trial. Lancet 2000;355:863-4.
8. Lasagna L. Diuretics vs alpha-blockers for treatment of hypertension: lessons from ALLHAT. Antihypertensive and Lipid-Lowering Treatment to Prevent Heart Attack Trial. JAMA 2000;283:2013-4.

9. Bradley HA, Wiysonge CS, Volmink JA, Mayosi BM, Opie LH. How strong is the evidence for use of beta-blockers as first-line therapy for hypertension? Systematic review and meta-analysis. J Hypertens 2006;24:2131-41

10. Lindholm LH, Carlberg B, Samuelsson O. Should beta blockers remain first choice in the treatment of primary hypertension? A meta-analysis. Lancet 2005;366:1545-53.

11. Wiysonge CS, Bradley $\mathbf{H}$, Mayosi BM, et al. Beta-blockers for hypertension. Cochrane Database Syst Rev 2007:CD002003.

12. Wright J, Musini V. First line drugs for hypertension. Cochrane Database Syst Rev 2009. Issue 3:Art. No.: CD00184110. doi:1002/ 14651858.CD001841.pub2.

13. Powers BJ, Coeytaux RR, Dolor RJ, et al. Updated Report on Comparative Effectiveness of ACE inhibitors, ARBs, and Direct Renin Inhibitors for Patients with Essential Hypertension: Much More Data, Little New Information. J Gen Intern Med 2012. doi:10.1007/s11606-011-1938-8.

14. Sanders G, Coeytaux R, Dolor R, et al. Angiotensin-Converting Enzyme Inhibitors (ACEIs), Angiotensin II Receptor Antagonists (ARBs), and Direct Renin Inhibitors for Treating Essential Hypertension: An Update. Comparative Effectiveness Review 2011; 34. http://www.effectivehealth care.ahrq.gov/ehc/products/164/695/CER-34-ACEIs-ARBs Executive-Summary_20110613.pdf. Accessed February 28, 2012.

15. Liberati A, Altman DG, Tetzlaff J, et al. The PRISMA statement for reporting systematic reviews and meta-analyses of studies that evaluate health care interventions: explanation and elaboration. Ann Intern Med 2009; 151:W65-94.

16. Moher D, Liberati A, Tetzlaff J, Altman DG. Preferred reporting items for systematic reviews and meta-analyses: the PRISMA statement. Ann Intern Med 2009;151:264-9

17. Dahlof B, Devereux RB, Kjeldsen SE, et al. Cardiovascular morbidity and mortality in the Losartan Intervention For Endpoint reduction in hypertension study (LIFE): a randomised trial against atenolol. Lancet 2002;359:995-1003.

18. Julius S, Kjeldsen SE, Weber $\mathbf{M}$, et al. Outcomes in hypertensive patients at high cardiovascular risk treated with regimens based on valsartan or amlodipine: the VALUE randomised trial. Lancet 2004;363:2022-31

19. Yusuf S, Teo KK, Pogue J, et al. Telmisartan, ramipril, or both in patients at high risk for vascular events. N Engl J Med 2008;358:1547-59.

20. Yusuf S, Teo $\mathbf{K}$, Anderson $\mathbf{C}$, et al. Effects of the angiotensin-receptor blocker telmisartan on cardiovascular events in high-risk patients intolerant to angiotensin-converting enzyme inhibitors: a randomised controlled trial. Lancet 2008;372:1174-83.

21. McMurray JJ, Holman RR, Haffner SM, et al. Effect of valsartan on the incidence of diabetes and cardiovascular events. N Engl $\mathrm{J}$ Med 2010;362:1477-90.

22. Yusuf S, Diener HC, Sacco RL, et al. Telmisartan to prevent recurrent stroke and cardiovascular events. N Engl J Med 2008;359:1225-37.

23. Bangalore S, Kumar S, Wetterslev J, Messerli FH. Angiotensin receptor blockers and risk of myocardial infarction: meta-analyses and trial sequential analyses of 147020 patients from randomised trials. BMJ 2011:342:d2234.

24. The Medical Letter Online. In brief: aliskiren trial terminated. 2012; 13825. 